\title{
Histologic criteria for diagnosing primary cutaneous malignant melanoma
}

\author{
Bruce R Smoller \\ Department of Pathology, University of Arkansas for Medical Sciences, Little Rock, AR, USA
}

\begin{abstract}
Malignant melanoma accounts for the largest number of deaths attributed to skin cancer. It also provides the most diagnostic challenges for the histopathologist. This article, attempts to describe the histologic features most closely associated with the various growth patterns of the most common subtypes of melanoma. While it has been shown repeatedly that histologic subtypes likely provide clinicians and patients with minimal to no prognostic information, it is useful to separate these entities in order to elucidate the varied histologic features seen within the class of tumors known as melanoma. The discussion centers around a checklist of changes seen at the microscope that are associated with this diagnosis. The goal of this chapter is to provide the reader with one perspective on the series of changes that are used in order to establish (or exclude) a diagnosis of melanoma. There is a comprehensive literature that critically evaluates histologic parameters associated with this collection of tumors and relates them to prognostic information, and no attempt will be made to correlate the histologic change with prognostic information. This will be discussed in another chapter in this volume. Similarly, more esoteric subtypes of melanoma are characterized by histologic features that differ from the common types of melanoma and will be addressed in another chapter.

Modern Pathology (2006) 19, S34-S40. doi:10.1038/modpathol.3800508
\end{abstract}

Keywords: melanoma; lentigo maligna; histology; prognosis

Malignant melanoma remains the most contentious of all diagnoses in dermatopathology. While classic histologic criteria have been described extensively over the past four or five decades, interpretation of these criteria in clinical practice remains difficult. This chapter will lay out and discuss many of the diagnostic criteria that are useful in practice. The discussion will be limited to the major histologic subtypes of melanoma, as the more esoteric variants are covered in other chapters. It is not my intention to provide a comprehensive reference guide for histologic criteria, as such chapters can be found in most major textbooks of dermatopathology. Rather, the series of observations that are most useful when attempting to arrive at such a diagnosis will be covered. Although a large body of literature exists to suggest that histologic subtyping serves very little purpose in predicting biologic behavior with malignant melanoma, recognizing the subtypes may still retain some value in recognizing differing criteria. ${ }^{1-6}$

The most common histologic pattern is the superficial spreading melanoma. These neoplasms repre-

Correspondence: Dr BR Smoller, MD, Department of Pathology, University of Arkansas for Medical Sciences, 4300 W. Markham, Little Rock, AR 72205, USA.

E-mail: smollerbrucer@uams.edu

Received 13 September 2005; accepted 28 September 2005 sent as many as $75 \%$ of all melanomas. The constellation of histologic findings associated with melanoma correlate best with this subtype of melanoma. Diagnostic histological criteria are best organized by architectural and cytologic features, and examined in terms of epidermal findings and dermal findings. Architectural changes seen within the epidermis in superficial spreading melanomas include poor circumscription of melanocytes, single melanocytes predominating over nests of melanocytes, haphazard and aberrant distribution of melanocytes, the presence of melanocytes above the basal layer (Pagetoid spread) and dyscohesive nests of melanocytes.

This subtype of melanoma is characterized by the lateral spreading of malignant melanocytes within the epidermis. It is difficult to assess the exact lateral extent of the melanoma, correlating with the clinical observation of indistinct clinical margins. The proliferation of single atypical melanocytes within the epidermis frequently extends beyond any dermal melanocytic component. In contrast, a benign melanocytic nevus demonstrates very sharp lateral margins.

In superficial spreading melanomas, abundant single melanocytes are present within the epidermis (Figure 1). These single melanocytes may be distributed as runs of cells along the dermal epidermal 


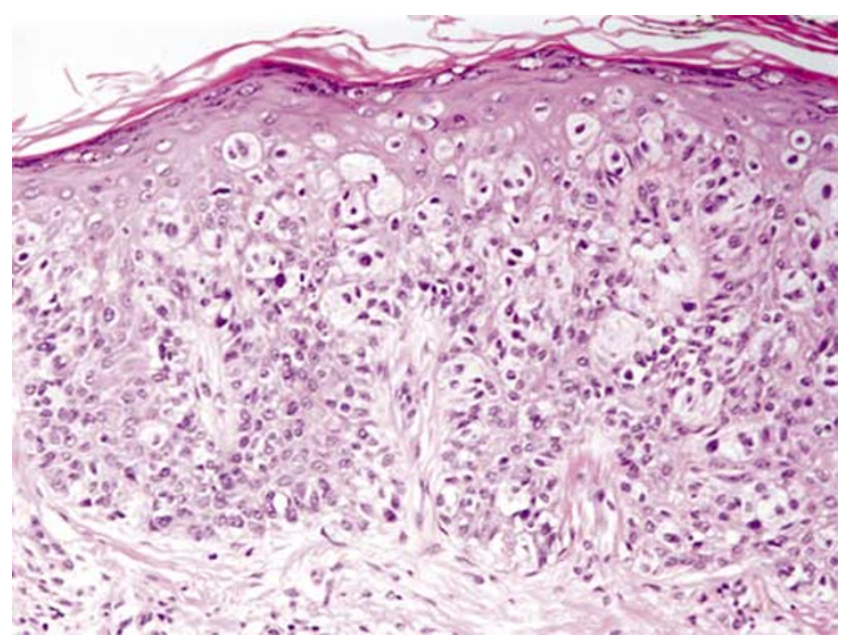

Figure 1 Superficial spreading melanoma with haphazardly distributed atypical melanocytes present as single cells and nests at all levels of the epidermis.

junction and commonly will be observed within the mid-portion and upper levels of the epidermis, as well. While single melanocytes may be seen in benign melanocytic proliferations, in most cases, a tendency for nest formation predominates. Some melanocytic proliferations demonstrate lentiginous basilar melanocytic hyperplasia, but in these cases, there is minimal cytologic atypia (see below) and Pagetoid extension is not present.

Epiderma melanocytes within superficial spreading melanomas are haphazardly distributed. It is not uncommon to observe runs of single cells immediately adjacent to large nests that are in direct proximity to smaller nests of melanocytes. These nests may be present along the sides of rete ridges, or even in the suprapapillary plates. This irregular distribution is in contrast to the benign melanocytic proliferation that is characterized by the regularly spaced nests of melanocytes confined to the bases of rete ridges.

Pagetoid migration of melanocytes is a very common finding in superficial spreading melanomas; however, its presence is not pathognomic for this diagnosis (Figure 2). It is commonly believed that these melanocytes located above the basal layer are not actively migrating, but rather, are physically disrupted from the basal lamina and are passively eliminated as the epidermis matures. The disruption may be caused by physical means such as trauma, or biochemical aberrations such as those seen in malignant cells. In superficial spreading melanomas, the Pagetoid distribution of melanocytes tends to spread over the breadth of the lesion, while it is more focal in benign melanocytic proliferations. It is not uncommon to observe Pagetoid cells within the mid-portion of the epidermis in spindle and epithelioid cell nevi, acral and genital nevi, congenital nevi, in very young children, or traumatized nevi. In each of these cases, however, the Pagetoid

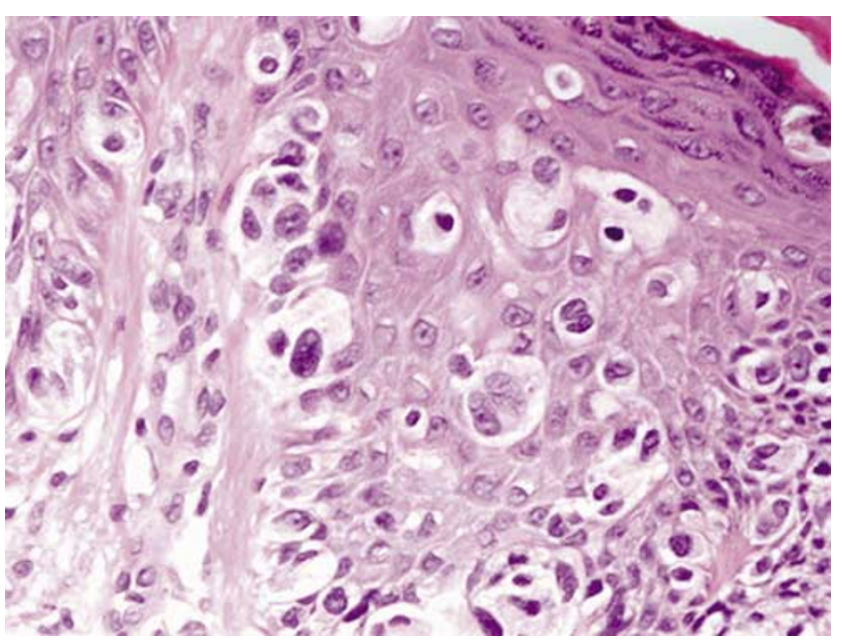

Figure 2 Abundant Pagetoid cells are present at all levels of the epidermis in melanoma.

cells are located in the central portions of the lesion and are relatively focal. Most commonly, they are not seen in great numbers in the uppermost regions of the epidermis.

A final low-power observation that is seen within the epidermal component of superficial spreading melanomas is dyscohesion within nests of melanocytes. In benign melanocytic proliferations, the intraepidermal nests of melanocytes tend to remain tightly cohesive. In spindle and epithelioid nevi, the nests may demonstrate separation from the surrounding keratinocytes with readily apparent cleft formation, but the melanocytic nests remain tightly cohesive. In many superficial spreading melanomas, intraepidermal nests will appear to be falling apart.

Intraepidermal melanoma cells are most commonly large cells with abundant eosinophilic cytoplasm, vesicular nuclei and very large, eosinophilic nucleoli. Nucleoli may be multiple. The cells are pleomorphic and mitoses are frequently found. Less commonly, the malignant cells may be small, and markedly hyperchromatic, and may display only scant amounts of cytoplasm (small nevoid melanoma). A spindle-cell morphology is unusual in this subtype of melanoma.

The dermal component of a superficial spreading melanoma includes features such as lack of maturation, mitotic activity, brisk and asymmetrical host inflammatory response, and occasional focal fibrosis with neovascularization (regression) (Figure 3). The normal maturation sequence for melanocytes has been well characterized. As melanocytes descend into the papillary dermis, they gradually diminish in size, demonstrate less apparent cytoplasm, less vesicular nuclei and inconspicuous nucleoli. Melanocytes at the base of ordinary nevi generally resemble lymphocytes (although they are somewhat larger). In superficial spreading melanomas, this maturation sequence is abortive or unapparent. The cells at the deepest extent of the dermal invasion 
S36

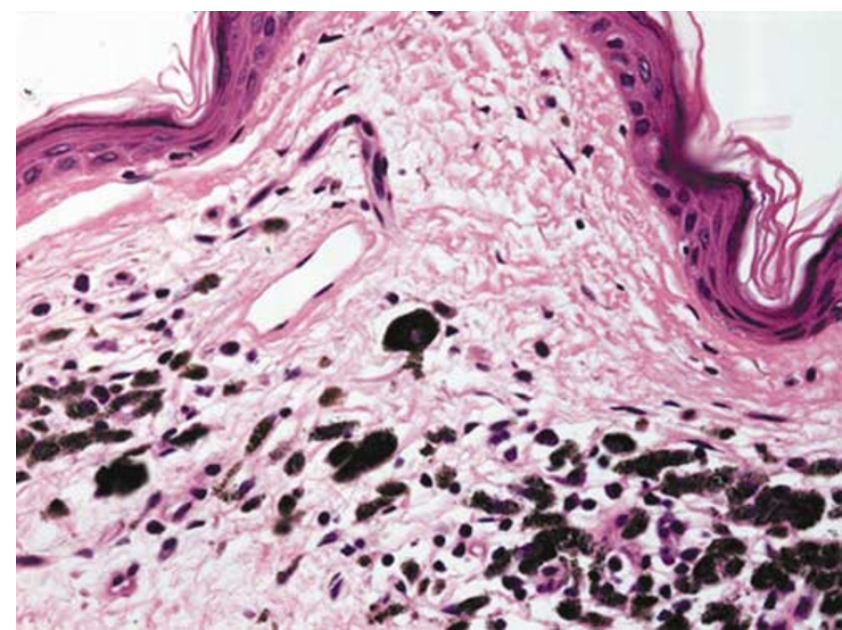

Figure 3 Regression is frequently seen within a melanoma and is characterized by loss of intraepidermal melanocytes, effacement of rete ridges, neovascularization, wispy fibrosis and a dense infiltrate of lymphocytes and melanophages.

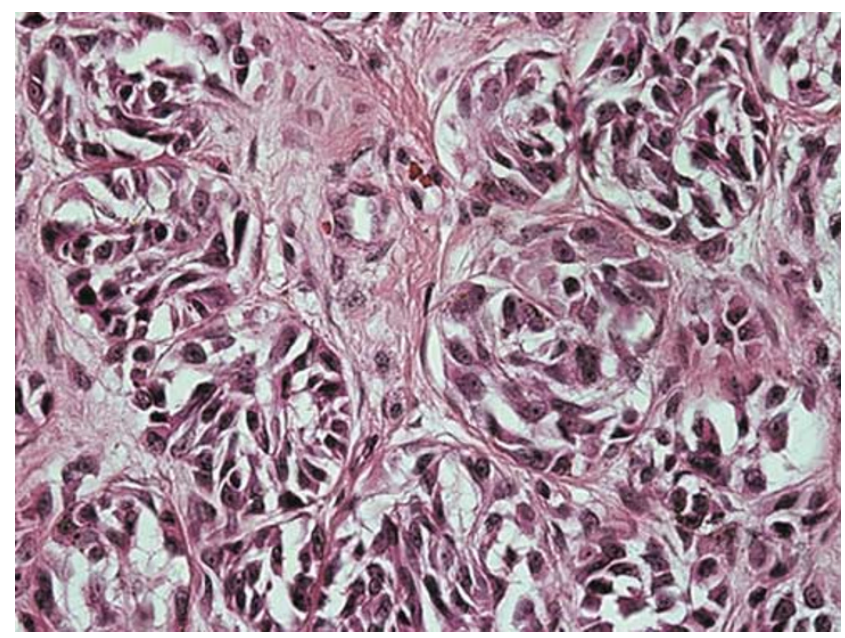

Figure 4 Even deep within the reticular dermis, these melanoma cells demonstrate vesicular nuclei and prominent nucleoli, evidence of lack of full maturation.

may be indistinguishable cytologically from those within the superficial papillary dermis (Figure 4). When assessing maturation, it is essential not to overinterpret maturation defects in melanocytes tracking down the adventitial collagen surrounding cutaneous appendages. In concert with individual melanocytes becoming smaller with progressive descent, the nesting pattern of these cells also changes reproducibly within benign nevi. Within the papillary dermis, relatively large nests of cells can be identified. As the nevus extends into the deeper dermis, the nests become smaller and, eventually, single melanocytes are found coursing between the reticular dermal collagen bundles.

The inflammatory response may be helpful in supporting a diagnosis of melanoma. An asymmetrical inflammatory infiltrate is often seen surround-

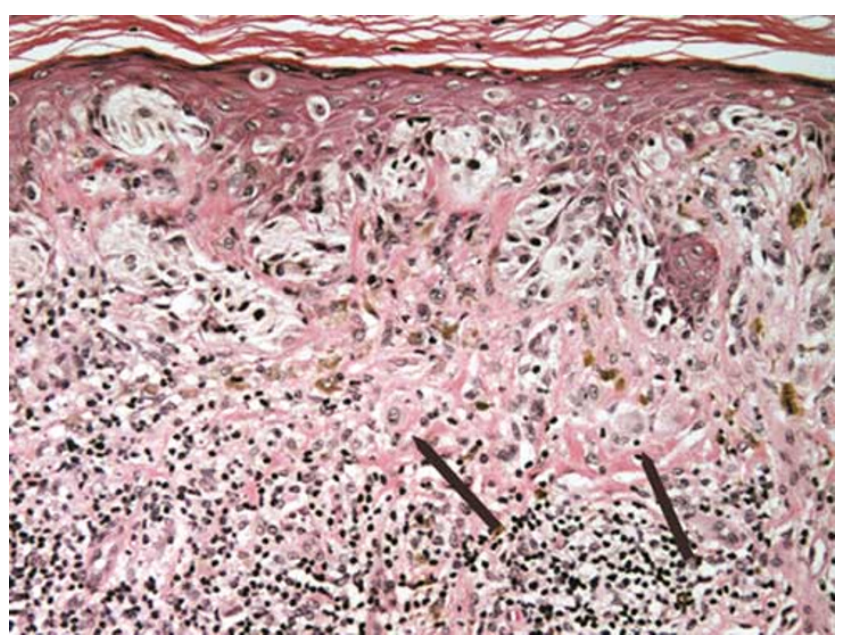

Figure 5 A brisk host response is present underlying a small focus of dermal invasion in this superficial spreading type of melanoma.

ing the invasive melanomas, but is unusual in benign melanocytic proliferations (Figure 5). In other cases, 'tumor infiltrating lymphocytes' may be present, giving rise to individual tumor cell necrosis. In these cases, it may be difficult to distinguish a melanoma from a halo nevus (that will not have the other histologic features of melanoma). Regression is also present in many invasive melanomas. Regression is defined as a partial host response to a malignant neoplasm, resulting in focal diminution of the process. Histologically, the changes are similar to those seen in a scar. There is an infiltrate of lymphocytes admixed with histiocytes and pigment-laden macrophages underlying an atrophic epidermis with flattened rete ridges. Nests of melanocytes are conspicuously absent from the epidermis overlying this dermal process. Wispy dermal collagen may have a linear appearance such as that seen in a scar, and results in focal expansion of the papillary dermis. There is an increase in dermal blood vessels, some of which may be ecstatic.

Another relatively common subtype of melanoma is the nodular melanoma. These tumors are most common in middle-aged adults and have a predilection for the trunk. Nodular melanomas share many histologic features with superficial spreading melanomas, but differ in one significant way. Unlike the situation in most superficial spreading melanomas, nodular melanomas do not demonstrate poor lateral circumscription (Figure 6a and b). In fact, these tumors are very sharply circumscribed. Further, the epidermal component does not extend laterally in relationship to the dermal component. Rather, the sharp demarcation within the epidermis parallels the dermal extension of the tumor. This correlates with the nodular clinical appearance of these neoplasms, and also with the observation that these tumors appear to have a worse prognosis. When 

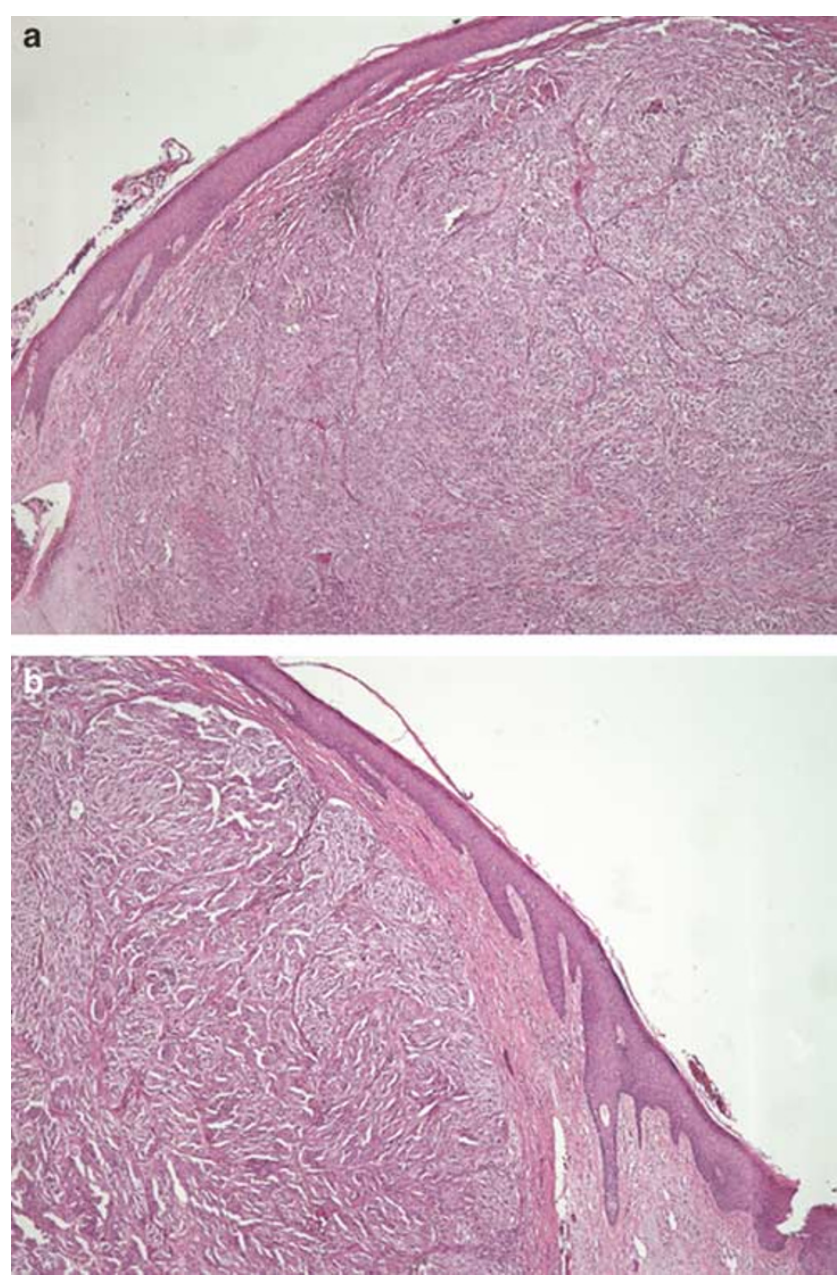

Figure 6 (a and b) These two photomicrographs demonstrate the sharp circumscription that characterizes nodular melanoma. A large, well-circumscribed proliferation of atypical melanocytes is seen growing throughout the dermis.

compared with superficial spreading melanomas, the prognosis is identical when controlled for depth of invasion; however, nodular melanomas do not have a radial growth phase, unlike superficial spreading melanomas. Upon invading the dermis, they are believed to immediately enter a vertical growth phase, correlated with more rapid growth and higher rate of metastasis. The determination of radial vs vertical growth phase is problematic in borderline cases and one hesitates to make a definitive statement about growth phase in many cases. In most nodular melanomas, however, the aggressive downward growth is apparent from the huge dermal nests and sheets of cytologically atypical melanocytes. Within the epidermal component, nodular melanomas are characterized by epithelioid melanocytes with abundant cytoplasm, vesicular nuclei and prominent nucleoli. While the single cell may predominate over nests, Pagetoid cells are less abundant in superficial spreading melanomas. Ulceration is commonly seen in these neoplasms.

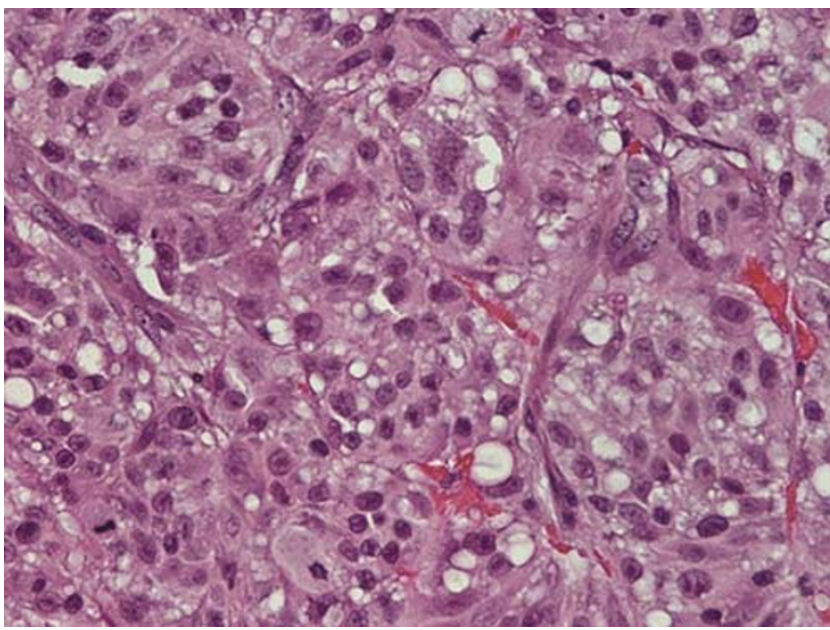

Figure 7 The dermal component of a nodular melanoma is characterized by markedly atypical, usually epithelioid melanocytes with lack of maturation and often a brisk mitotic activity.

The dermal component of nodular melanoma is histologically indistinguishable from superficial spreading melanoma that has entered the vertical growth phase. (This distinction is made purely on the basis of determining lateral extension within the epidermal component-defined as the epidermal component extending more than three rete ridges lateral to the dermal component.) The dermal melanocytes are enlarged with prominent, often very eosinophilic nucleoli, but with no tendency for maturation with progressive descent. Mitotic activity ranges from brisk to inconspicuous (Figure 7). Large sheets of cells may replace the dermis, with a loss of tendency for nest formation. Regression is often seen within these lesions, and the host response varies from brisk to nonexistent. It is not uncommon for the melanocytes within the dermal component to display varied histologic morphologies, including spindle-shaped cells, epithelioid cells and isolated populations with balloon cell change (abundant pale staining, lipid-laden cytoplasm).

In most cases, the diagnosis of this subtype of melanoma is not difficult owing to the extensive dermal component and the marked atypia that characterizes the malignant cells. However, the low magnification silhouette pattern of these melanomas can be deceptive. By definition, there is no lateral extension of the intraepidermal component, giving the tumor a well circumscribed, often symmetrical architectural pattern. When the cytologic features are more subtle (as in small nevoid, or minimal deviation melanoma, described elsewhere in this volume), the diagnosis can become difficult. Attention to such features as true, complete maturation, dermal mitotic activity and focal inflammatory host responses can be helpful in arriving at the correct diagnosis.

Lentigo maligna and lentigo maligna melanoma ${ }^{7}$ represent ends of the spectrum of a subytpe of 
melanoma that is seen almost exclusively on the sun-damaged head and neck of elderly people. Its incidence is not known, but appears to be rising sharply. Lentigo maligna is the in situ phase of lentigo maligna melanoma. (In order to diminish confusion over nomenclature, these lesions are diagnosed as 'melanoma in situ, lentigo maligna type'.) The intraepidermal component demonstrates several features that are distinct from the subtypes previously described. By definition, the epidermis is atrophic in this type of melanoma that only occurs on extensively sun-damaged skin. Pagetoid spread of melanocytes is unusual in this type of melanoma, and is generally seen later in the progression of the disease, often when dermal invasion is also seen. The more usual pattern is to find confluent melanocytes along the dermal epidermal junction, frequently extending deep into the appendageal epithelium. The cells are most commonly hyperchromatic and small. Nuclear chromatin is dense and nucleoli are often unapparent (Figure 8). Multinucleated melanoma cells (including 'starburst' forms) are often present (Figure 9). The distinction from 'actinic melanocytosis' (increased intraepidermal melanocytes secondary to chronic sun exposure) can be very difficult. This benign, reactive condition is also characterized by increased numbers of single basilar melanocytes occurring in the setting of an atrophic epidermis. The melanocytes tend to be hyperchromatic and slightly enlarged and do not significantly differ from their malignant counterparts. The main distinguishing features are numbers of cells (more in melanoma in situ), presence of Pagetoid extension (when present), and extension down cutaneous appendages (not a pathognomonic feature).

Lentigo maligna melanoma is, by definition, a melanoma that invades the dermis. In this subtype

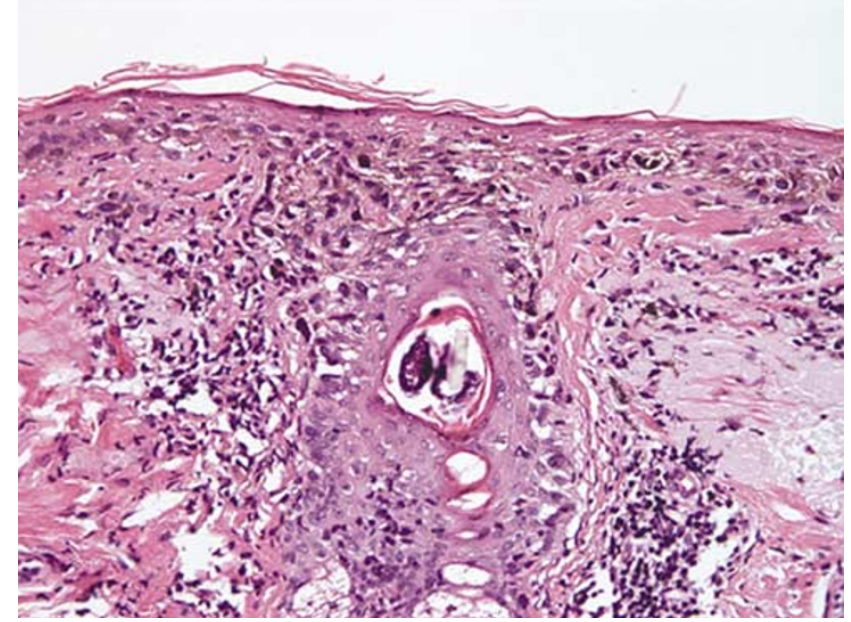

Figure 8 Lentigo maligna is characterized by confluent single melanocytes aligned along the dermal epidermal junction and spreading down cutaneous appendages. The cells are small and hyperchromatic and Pagetoid extension is uncommon. There is always underlying solar elastosis. of melanoma, the dermis is invariably characterized by marked solar elastosis. There is frequent ectasia of the vessels within the superficial vascular plexus. Atypical melanocytes are present as nests and single cells within the dermis. The dermal melanocytes most commonly display a spindle-shaped morphology, although epithelioid cells can be seen. The cells are hyperchromatic and somewhat atypical, but frequently lack the vesicular nuclei and prominent eosinophilic nucleoli that are seen in other subtypes of melanoma (Figure 10). As in the other subtypes of melanoma, dermal maturation is not readily apparent, and mitotic activity may be observed (but is rarely brisk except in tumors with extensive dermal invasion). The spindle-shaped melanocytes have a predilection for nerves within the reticular dermis, and perineural invasion is often seen. In these cases, prominent nerves may be a helpful clue (Figure 11). The melanoma cells can be found coursing individually through the dermis, or seen in concert with a stromal proliferation. The stroma may be mucinous with varying degrees of cellularity, or relatively sclerotic. In some cases (desmoplastic melanoma, described elsewhere in this volume) the invading tumor cells may be very subtle, bearing a striking resemblance to a dermal scar.

Acral lentiginous melanoma is a relatively rare subtype of melanoma that is found on acral surfaces. These tumors often arise within nail beds, under the nail plates, and thus present late in the course of the disease. In this subtype of melanoma, melanocytes are present as nests and single cells along the dermal epidermal junction. Pagetoid upward migration tends to be extensive and widespread over the breadth of these lesions. Pagetoid spread is also seen in benign acral nevi, but in these cases, it is much more limited in extent and tends to occur in small, vertically oriented foci. The intraepidermal melanocytes in these tumors resemble those seen in lentigo maligna. The melanocytes are most com-

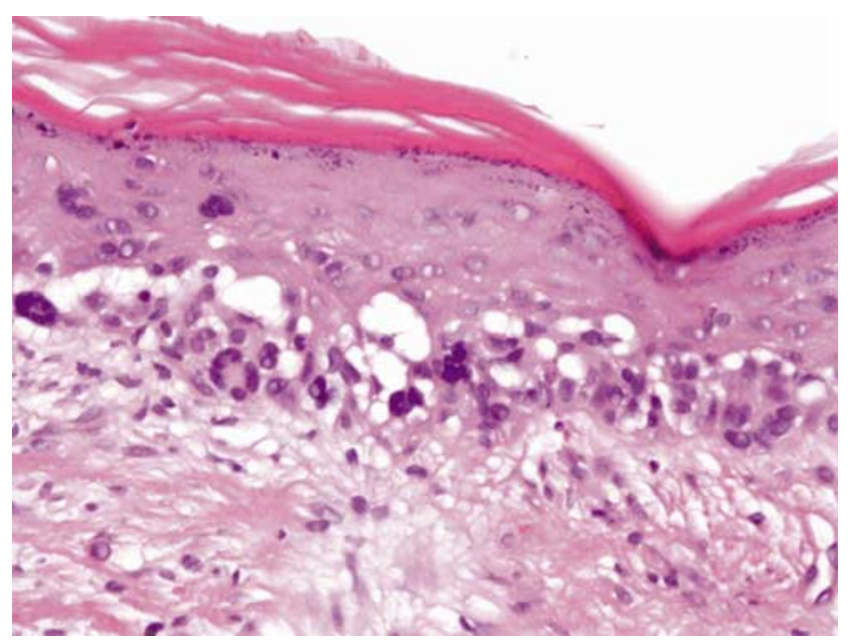

Figure 9 Lentigo maligna demonstrating multinucleated (starburst) cells. 


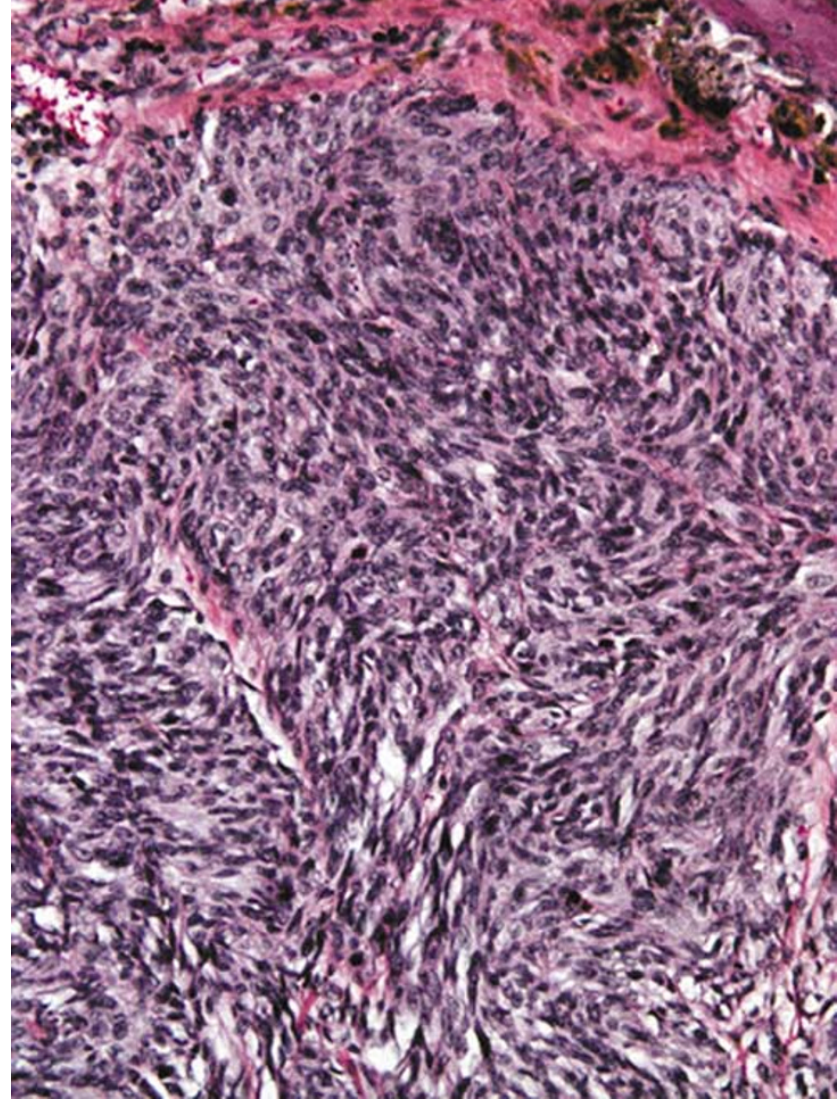

Figure 10 The dermal component of lentigo maligna melanoma is often characterized by a proliferation of spindle-shaped, hyperchromatic melanocytes that may lack pigment production.

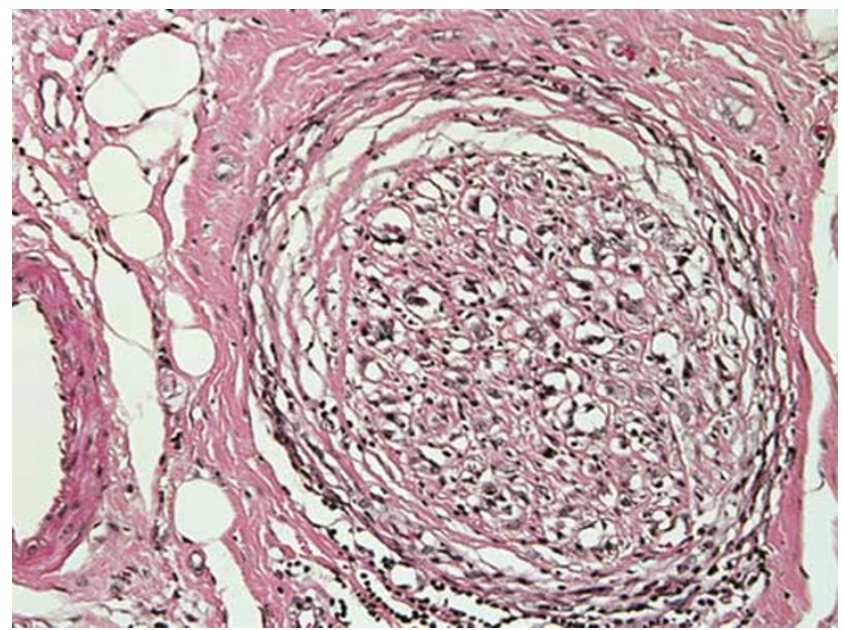

Figure 11 Atypical melanocytes are coursing around this markedly enlarged nerve within the reticular dermis in a lentigo maligna melanoma.

monly hyperchromatic and somewhat spindled in configuration. Nucleoli are not readily apparent in many cases (Figure 12).

Dermal invasion is characterized by a proliferation of spindle shaped, hyperchromatic melanocytes

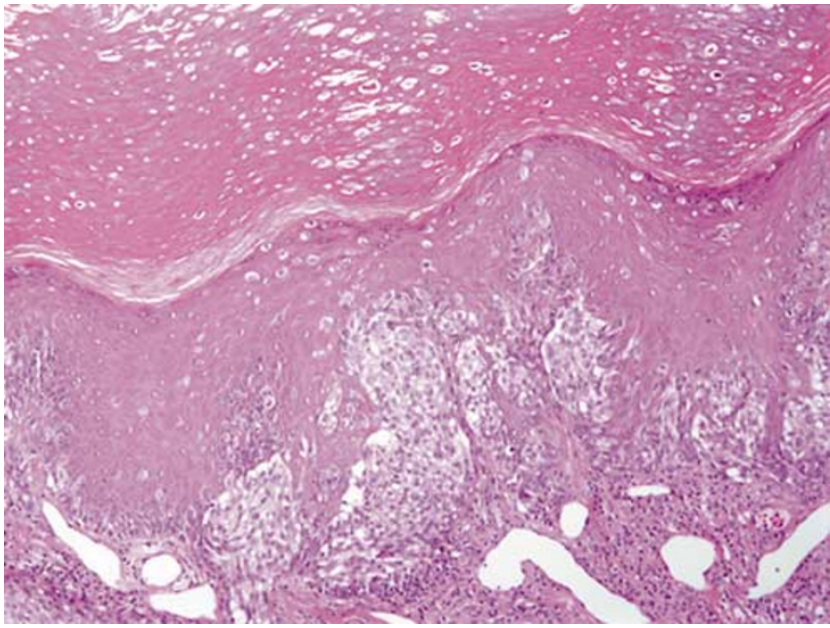

Figure 12 Acral lentiginous melanoma demonstrates nests and single atypical melanocytes within the epidermis with extensive Pagetoid upward migration.

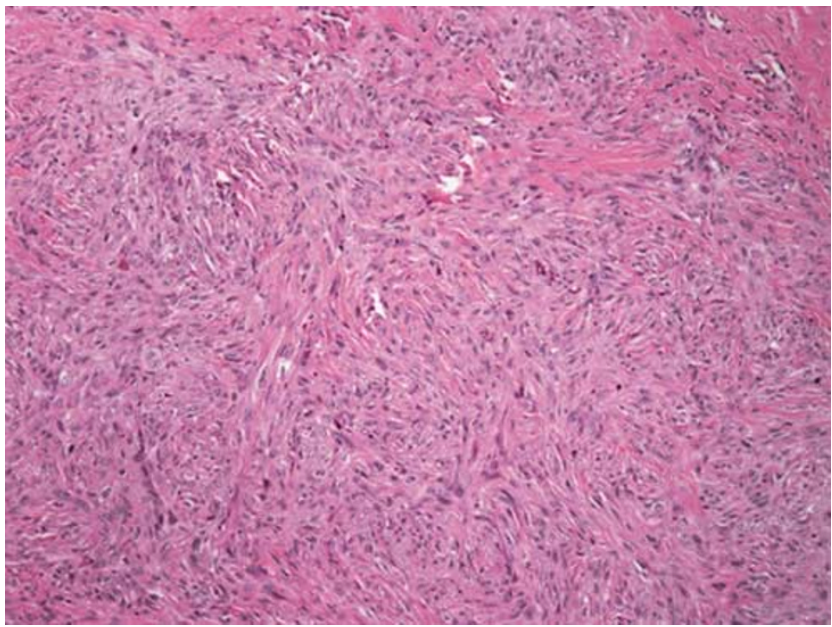

Figure 13 The dermal component of acral lentiginous melanoma generally demonstrates fascicles of spindle-shaped melanocytes that may course within fibrotic stroma.

coursing in fascicles, nests and single cells through the dermis. There is a marked tendency to track down eccrine structures, and aggregation around blood vessels is often seen. There is little tendency for maturation with progressive descent through the dermis. Mitotic activity is variable in degree (Figure 13). Mucosal lentiginous melanomas share many of the histologic characteristics of acral lentiginous melanomas and differ mainly in the underlying anatomic sites involved. Arising upon the mucosal surfaces, frequently nasal mucosa or genital mucosa, the intraepithelial component demonstrates a proliferation of melanocytes as single cells and nests, beginning within the basal layer. The mucosal surface is often spongiotic and may be acanthotic. Ulceration is frequently encountered. The intraepithelial component of this melanocytic proliferation is characterized by one of the two earlier morphologic characteristics. In some cases, the cells 
S40

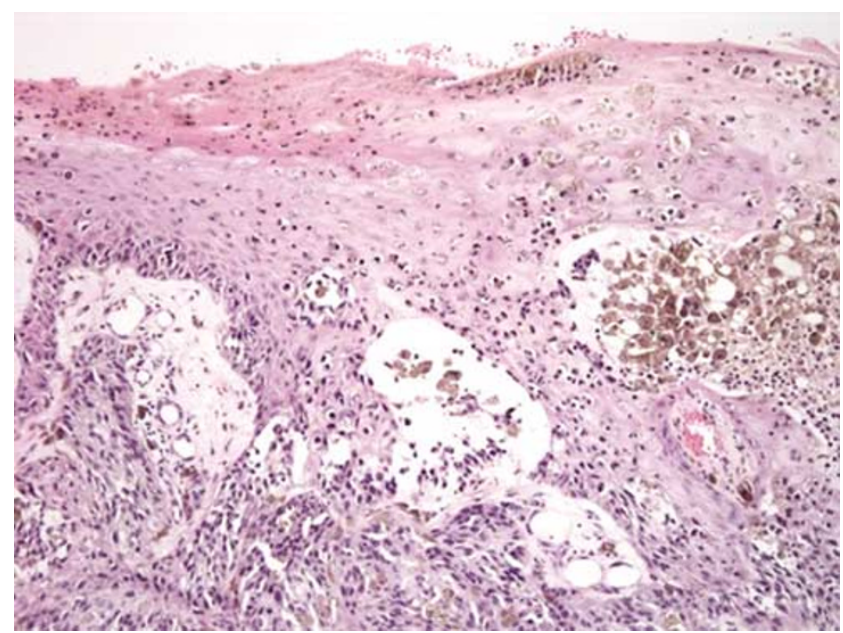

Figure 14 Mucosal lentiginous melanoma demonstrates large nests and single atypical melanocytes at all levels of the mucosa surface, often with ulceration.

are large and epithelioid, with abundant eosinophilic cytoplasm. In these cases, prominent and enlarged nucleoli may be seen. In other cases, the melanocytes are enlarged, with hyperchromatic and spindle-shaped nuclei and relatively inconspicuous amounts of cytoplasm. Nucleoli are often absent (Figure 14).

The invasive component of mucosal lentiginous melanomas is similar to that seen in acral lentiginous and lentigo maligna melanomas. Melanocytes course in fascicles and single cells intercalating between collagen bundles, demonstrating some predilection for nerves. The neoplastic cells often have a spindle-shaped morphology and are accompanied by a myxoid or desmoplastic stromal response. Scattered mitoses may be seen and there is little tendency for maturation with progressive descent. Cytologic atypia ranges from slight (unusual) to marked.

\section{References}

1 Clark WH, Elder DE, Van Horn M. The biologic forms of malignant melanoma. Hum Pathol 1986;17:443-450.

2 Ackerman AB, David KM. A unifying concept of malignant melanoma: biologic aspects. Hum Pathol 1986;17:438-442.

3 Mihm Jr MC, Clark Jr WH, From L. The clinical diagnosis, classification and histogenetic concepts of the early stages of cutaneous malignant melanomas. $\mathrm{N}$ Engl J Med 1971;284:1078-1082.

4 Clark WH, Elder DE, Guerry IV D, et al. A study of tumor progression: the precursor lesions of superficial spreading and nodular melanoma. Hum Pathol 1984;12:11471165.

5 Sober AJ, Fitzpatrick TB, Mihm Jr MC. Primary melanoma of the skin: recognition and management. J Am Acad Dermatol 1980;2:179-197.

6 Cochran AJ, Bailly C, Cook M, et al. Recommendations for the reporting of tissues removed as part of the surgical treatment of cutaneous melanoma. Association of Directors of Anatomic and Surgical Pathology. Hum Pathol 1997;28:1123-1125.

7 Flotte TJ, Mihm Jr MC. Lentigo maligna and malignant melanoma in situ, lentigo maligna type. Hum Pathol 1999;30:533-536. 